\title{
Efficient and Stable Large-Area Perovskite Solar Cells with Inorganic Perovskite/Carbon Quantum Dot-Graded Heterojunction
}

\author{
Qiang Sun, ${ }^{1}$ Cai Shen $\left(\mathbb{D},{ }^{2}\right.$ Deyu Wang, ${ }^{2}$ Tao Zhang, ${ }^{1}$ Huaxia Ban, ${ }^{1}$ Yan Shen, \\ Zhipan Zhang, ${ }^{3}$ Xiao-Li Zhang, ${ }^{4}$ Guanjun Yang, ${ }^{5}$ and Mingkui Wang ${ }^{1}$ \\ ${ }^{1}$ Wuhan National Laboratory for Optoelectronics, Huazhong University of Science and Technology, Wuhan, Hubei 430074, China \\ ${ }^{2}$ Ningbo Institute of Materials Technology \& Engineering, Chinese Academy of Sciences, 1219 Zhongguan Road, \\ Ningbo 315201, China \\ ${ }^{3}$ School of Chemistry, Beijing Institute of Technology, Beijing 102488, China \\ ${ }^{4}$ State Centre for International Cooperation on Designer Low-Carbon \& Environmental Materials, School of Materials Science \\ and Engineering, Zhengzhou University, 450001, China \\ ${ }^{5}$ State Key Laboratory for Mechanical Behavior of Materials, Xi'an Jiaotong University, Xi'an 710049, China
}

Correspondence should be addressed to Mingkui Wang; mingkui.wang@mail.hust.edu.cn

Received 27 March 2021; Accepted 4 June 2021; Published 12 July 2021

Copyright (c) 2021 Qiang Sun et al. Exclusive Licensee Science and Technology Review Publishing House. Distributed under a Creative Commons Attribution License (CC BY 4.0).

\begin{abstract}
This work reports on a compositionally graded heterojunction for photovoltaic application by cooperating fluorine-doped carbon quantum dots (FCQDs in short) into the $\mathrm{CsPbI}_{2.5} \mathrm{Br}_{0.5}$ inorganic perovskite layer. Using this $\mathrm{CsPbI}{ }_{2.5} \mathrm{Br}_{0.5} / \mathrm{FCQDs}$ graded heterojunction in conjunction with low-temperature-processed carbon electrode, a power conversion efficiency of $13.53 \%$ for $1 \mathrm{~cm}^{2}$ all-inorganic perovskite solar cell can be achieved at AM 1.5G solar irradiation. To the best of our knowledge, this is one of the highest efficiency reported for carbon electrode based all-inorganic perovskite solar cells so far, and the first report of $1 \mathrm{~cm}^{2}$ carbon counter electrode based inorganic perovskite solar cell with PCE exceeding 13\%. Moreover, the inorganic perovskite/carbon quantum dot graded heterojunction photovoltaics maintained over $90 \%$ of their initial efficiency after thermal aging at $85^{\circ}$ for 1056 hours. This conception of constructing inorganic perovskite/FCQDs graded heterojunction offers a feasible pathway to develop efficient and stable photovoltaics for scale-up and practical applications.
\end{abstract}

\section{Introduction}

Lead halide perovskites with the $\mathrm{ABX}_{3}$ formula (where $\mathrm{X}$ is halogen anion, $\mathrm{A}$ is monovalent cation (methylammonium $\left(\mathrm{MA}^{+}\right)$, formamidinium $\left(\mathrm{FA}^{+}\right)$or $\left.\mathrm{Cs}^{+}\right)$, and $\mathrm{B}$ is $\mathrm{Pb}^{2+}$ ) have emerged as disruptive photovoltaic materials for solar energy conversion owning to a rapid increase in power conversion efficiency (PCE) [1-5]. However, the instability against thermal stress and humidity originated from the hygroscopic and volatile nature of organic cations (including $\mathrm{MA}^{+}$and $\mathrm{FA}^{+}$) in these organic-inorganic hybrid perovskites as well as organic materials for collecting currents in a complete device has been the primary issue to hinder their commercialization [6-8]. Therefore, a solar cell free of organic components, which is constructed solely with inorganic perovskite com- pounds and inorganic functional materials including charge transport materials and contact electrode materials, offers great possibilities of enhancing solar cells performance with high stability.

In this regard, all-inorganic perovskite solar cells (PSCs) with carbon counter electrodes are thus developed in view of the structural stability and cost-effectiveness, and have become research interests arising from great prospect toward commercialization [9-14]. For example, an all-inorganic PSC with a structure of $\mathrm{FTO} / \mathrm{TiO}_{2} / \mathrm{CsPbIBr}{ }_{2} /$ carbon has been reported by Zhu et al., which achieved a PCE over $9 \%$ and an open-circuit voltage $\left(V_{\mathrm{OC}}\right)$ of $1.245 \mathrm{~V}$ [10]. Previously, we reported $10.8 \%$ efficient stable full-printable PSCs using an inorganic metal oxide framework of $\mathrm{FTO} / \mathrm{TiO}_{2} / \mathrm{Al}_{2} \mathrm{O}_{3} /$ $\mathrm{NiO} /$ carbon and inorganic perovskite $\mathrm{CsPb}_{0.98} \mathrm{Mg}_{0.02} \mathrm{I}_{2} \mathrm{Br}$ 
[15]. The inorganic perovskites featuring with excellent compositional and thermal stability offer promising potentials to process solar cells with high stability [16-18]. The utilization of moisture resistant carbon counter electrode offers additional advantages to inorganic PSCs, including good stability in ambient conditions and low-cost manufacture [19]. Moreover, the carbon electrode based PSCs can be fabricated with printing technology such as gravure printing and flexographic printing, which is beneficial for the scalable production of large modules [20]. Unfortunately, relatively lower PCE (typically 7\%-11\%) has been often observed for the inorganic PSCs using carbon counter electrode comparing to their counterparts that using $2,2^{\prime}, 7,7^{\prime}$-tetrakis $(N, N$-di-4methoxyphenylamino)-9, $9^{\prime}$-spiro-bifluorene (spiro-OMe$\mathrm{TAD}$ ) as the hole-transport layer (HTL) and Au as the counter electrode [19, 21].

The interface between perovskite layer and carbon electrode is crucial to the photovoltaic performance of carbon electrode based PSCs. A poor interfacial contact as well as mismatched energy level alignment between perovskite layer and carbon electrode significantly induces undesirable charge recombination [22-25]. This also explains the widely observed large $V_{\mathrm{OC}}$ loss (defined as $E_{g} / q-V_{\mathrm{OC}}$, where $E_{g}$ is the bandgap of the absorption material, $q$ is elementary charge), as well as low fill factor (FF) for carbon electrode based PSCs [9-14]. Considering the big resistance of carbon counter electrode $(\sim 20-40 \Omega / \square)$ with large-sized particles of high porosity, indeed, minimization of the interfacial recombination kinetics gives a good shot of enhancing $V_{\mathrm{OC}}$ along with FF. Therefore, in aim to enhance the photovoltaic performance of carbon electrode based all-inorganic PSCs, it is of fundamental importance to overcome the limits through the formation of intimate contact at the perovskite/carbon electrode interface and tailoring the interfacial energy-band alignment.

Graded-heterojunction (GHJ) engineering can be an effective method to facilitate the separation of photogenerated charge carriers and their transport in photovoltaic devices [26-30]. For example, an organic-inorganic hybrid perovskite-fullerene graded-heterojunction structure has been proposed by $\mathrm{Wu}$ and coworkers for the invertedstructured planar PSCs, which enabled the fabrication of centimeter-scale PSC device with a certified PCE exceeding 18\% [26]. The carbon electrode-based PSCs with $\mathrm{MAPbI}_{3}{ }^{-}$ PTAA gradient heterojunction also achieved a PCE of $13.0 \%$ in active area of $1 \mathrm{~cm}^{2}$ [29]. The graded-heterojunction architecture combines the advantage of short charge travel distance with the merit of introducing band slope at the heterojunction interface for transport of photo-generated charge carriers. Consequently, charge recombination loss can be minimized and thus augments the open-circuit voltage.

This work for the first time reports on compositionally graded heterojunction for photovoltaic application by injecting fluorine-doped carbon quantum dots (FCQDs in short) into inorganic $\mathrm{CsPb}_{2.5} \mathrm{Br}_{0.5}$ perovskite via the antisolvent method. Because of the excellent optoelectronic properties together with high chemical stability, carbon quantum dots have shown significant potential as charge transport material or surface passivating agent in photovoltaic devices [31-34]. In this case, due to its small size and high polarization property, FCQDs carried by antisolvent (chlorobenzene) can be deposited into the $\mathrm{CsPb}_{2.5} \mathrm{Br}_{0.5}$ perovskite layer before perovskite crystallization, forming a perovskite/ FCQDs graded heterojunction with increasing FCQDs percentage along the vertical direction. The results indicate that the FCQDs interact strongly with $\mathrm{CsPbI}_{2.5} \mathrm{Br}_{0.5}$ perovskite and efficiently passivate the uncoordinated $\mathrm{Pb}$ ions defects. Moreover, the $\mathrm{CsPbI}_{2.5} \mathrm{Br}_{0.5} / \mathrm{FCQDs}$ graded heterojunction selectively promotes photo-generated charge separation and extraction, resulting in improved photovoltaic performance. The $\mathrm{CsPbI}_{2.5} \mathrm{Br}_{0.5} / \mathrm{FCQD}$-graded heterojunction in conjunction with low-temperature-processed carbon electrode achieved an impressive power conversion efficiency of $13.53 \%$ in a $1 \mathrm{~cm}^{2}$ device at AM $1.5 \mathrm{G}$ solar irradiation. More importantly, the solar cells based on $\mathrm{CsPbI}_{2.5} \mathrm{Br}_{0.5} / \mathrm{FCQDs}$ $\mathrm{GHJ}$ in combination with low-temperature processed carbon electrode exhibited excellent long-term stability. The unencapsulated devices maintained over $90 \%$ of their initial efficiency under thermal aging at $85^{\circ} \mathrm{C}$ for $1056 \mathrm{~h}$.

\section{Results}

The $\mathrm{CsPbI}_{2.5} \mathrm{Br}_{0.5} / \mathrm{FCQD}$ graded heterojunction was fabricated by injecting FCQDs into inorganic $\mathrm{CsPb}_{2.5} \mathrm{Br}_{0.5}$ perovskite layer via antisolvent method during perovskite precursor spin-coating process as illustrated in Figure 1(a). In this case, partial FCQDs in a size of about $3-7 \mathrm{~nm}$ (characterized with transmission electron microscopy, Figure S1) carried by antisolvent chlorobenzene (CB) can be deposited into the upper layer of $\mathrm{CsPb}_{2.5} \mathrm{Br}_{0.5}$ perovskite film before crystallization, while most of FCQDs maintain on perovskite layer surface, forming a $\mathrm{CsPb}_{2.5} \mathrm{Br}_{0.5} / \mathrm{FCQDs}$ graded heterojunction structure with increasing FCQDs percentage along the vertical direction. This GHJ conception was verified by time-of-flight secondary-ion mass spectrometry (TOF-SIMS) characterization as shown in Figure 1(b) [15, 35]. When sputtering through the $\mathrm{CsPbI}_{2.5} \mathrm{Br}_{0.5} / \mathrm{FCQDs} \mathrm{GHJ}$ thin film by using $\mathrm{Bi}^{3+}$ ion beam, the SIMS characterization detects the signals of $\mathrm{Pb}$, $\mathrm{I}, \mathrm{Br}$, and $\mathrm{C}$ in negative mass detection mode, in which $\mathrm{C}$ was used as an indicator of FCQDs. Obviously, the C depth profile (blue line) shows a steep decline at the perovskite/FCQDs GHJ film surface and negligible counting amount in the bulk. The decrease of carbon content along the vertical direction verifies a graded distribution of FCQDs in the $\mathrm{CsPbI}_{2.5} \mathrm{Br}_{0.5}$ perovskite absorption layer.

The interfacial band alignment around $\mathrm{CsPbI}_{2.5} \mathrm{Br}_{0.5}$ and FCQDs was estimated by ultraviolet photoelectron spectroscopy (UPS) and UV-visible absorption spectroscopy characterization (Figure S2a-S2c). The Fermi energy level $\left(E_{F}\right)$ of $\mathrm{CsPbI}_{2.5} \mathrm{Br}_{0.5}$ and FCQDs was calculated to be -4.36 and $-4.74 \mathrm{eV}$, respectively (Figure S2d). The corresponding valence band maximum $\left(E_{\mathrm{VB}}\right)$ of $\mathrm{CsPbI}_{2.5} \mathrm{Br}_{0.5}$ and FCQDs was further evaluated to be $-5.61 \mathrm{eV}$ and $-5.37 \mathrm{eV}$, respectively. By taking into account of the valance band maximum, the conduction band minimum, and the Fermi 


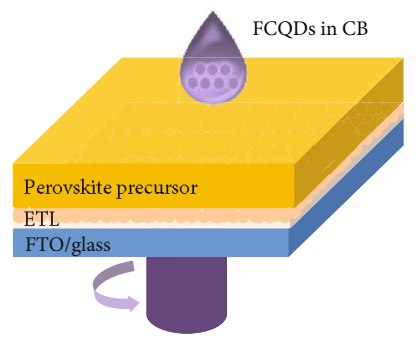

(a)

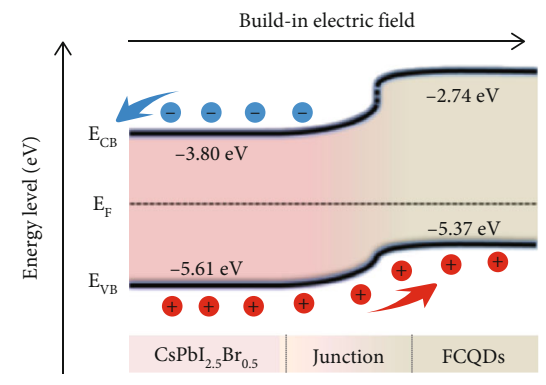

(c)
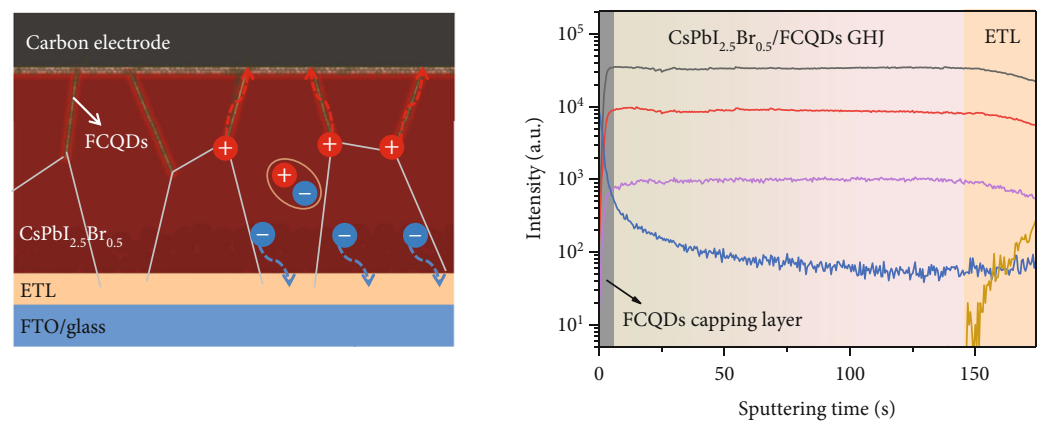

(b)
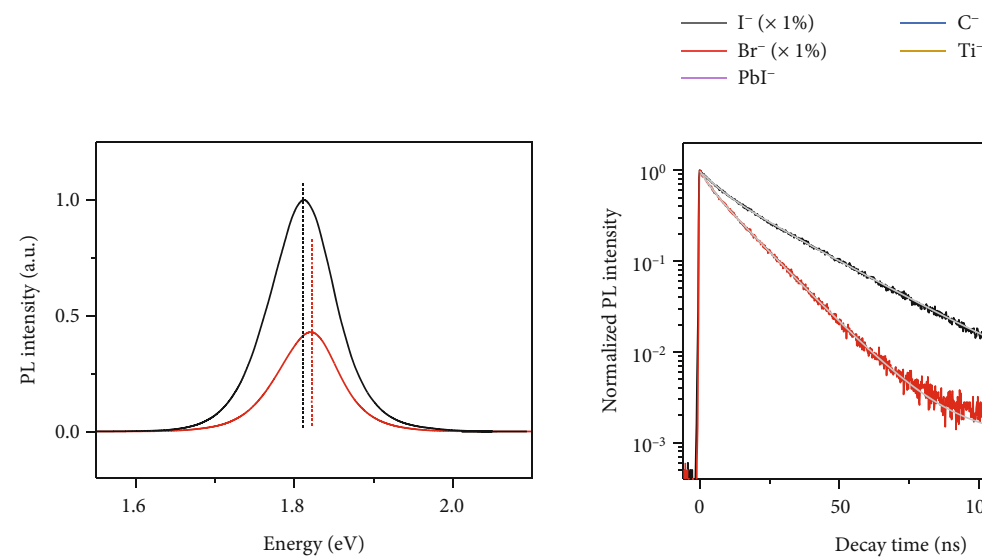

(d)

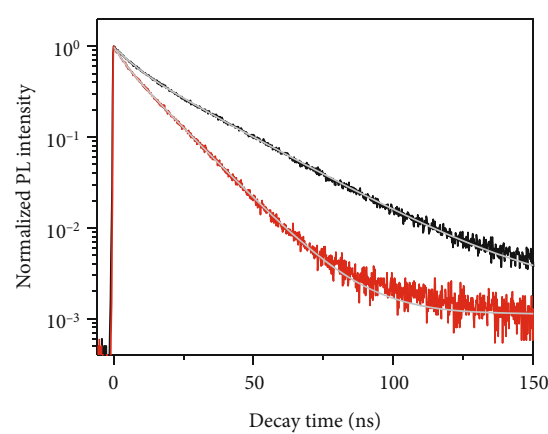

(e)

Control
- GHJ sample

FIgURE 1: Material characterization of the graded heterojunction. (a) Schematic diagram for preparation of $\mathrm{CsPb}_{2.5} \mathrm{Br}_{0.5} / \mathrm{FCQD} \mathrm{graded}$ heterojunction devices. (b) TOF-SIMS depth profiles of $\mathrm{CsPb}_{2.5} \mathrm{Br}_{0.5} / \mathrm{FCQDs}$ graded heterojunction thin film. (c) Schematic diagram of band bending at the $\mathrm{CsPbI}_{2.5} \mathrm{Br}_{0.5}$ perovskite/FCQDs interface. Steady-state photoluminescence spectra (d) and time-resolved photoluminescence decay (e) curves of the $\mathrm{CsPb}_{2.5} \mathrm{Br}_{0.5}$ perovskite thin film and perovskite/FCQDs GHJ thin film on glass substrates.

level of both $\mathrm{CsPbI}_{2.5} \mathrm{Br}_{0.5}$ and FCQDs, upon contact, electron is depleted from $\mathrm{CsPbI}_{2.5} \mathrm{Br}_{0.5}$ to FCQDs, causing an upward band bending at the $\mathrm{CsPb}_{2.5} \mathrm{Br}_{0.5} / \mathrm{FCQDs}$ interface. Base on this understanding, we built up an energy-level diagram for the perovskite/FCQDs GHJ in Figure 1(c), which schematically illustrates the upper shift valance band of $\mathrm{CsPbI}_{2.5} \mathrm{Br}_{0.5}$ at the heterojunction interface. In addition to concentration gradient, this kind of band bending supplies additional driving force for photogenerated hole extraction and restrains electron flowing back.

Figure S3 shows scanning electron microscopy (SEM) image of surface morphology of the as-prepared perovskite/ FCQDs GHJ thin film. The corresponding films are very dense and smooth with few aggregates and pinholes. The $\mathrm{X}$-ray photoelectron spectroscopy (XPS) characterization in Figure S4 presents the chemical states of the as-fabricated films. The binding energies of $\mathrm{Pb}\left(4 \mathrm{f}_{7 / 2}\right)$ and $\mathrm{Pb}\left(4 \mathrm{f}_{5 / 2}\right)$ at $138.2 \mathrm{eV}$ and $143.05 \mathrm{eV}$ for $\mathrm{CsPbI}_{2.5} \mathrm{Br}_{0.5}$ sample positively shift to higher values by $0.015 \mathrm{eV}$ for $\mathrm{CsPbI}_{2.5} \mathrm{Br}_{0.5} /$ FCQDs GHJ sample. This is usually caused with the existence of additional negative charges surrounding $\mathrm{Pb}$ ions [36-38]. In this work, we attributed this to the presence of fluorine in FCQDs, which provides extra electrons toward uncoordinated $\mathrm{Pb}$ ions at the perovskite film surface and grain boundaries, forming strong $\mathrm{Pb}-\mathrm{F}$ ionic bonds, therefore efficiently passivating the widespread uncoordinated $\mathrm{Pb}$ ions defects. Recently, $\mathrm{Li}$ et al. employed fluoride to simultaneously passivate both anion and cation vacancies of halide perovskites, by taking the advantage of its high electronegativity [39]. This conclusion agrees well with our previous reports that the incorporation of fluorinecontaining functional groups at the perovskite/HTL interface can efficiently passivate the interfacial defects and boost the photovoltaic performances of PSCs [40]. Figure 1(d) presents the results of steady-state photoluminescence $(\mathrm{PL})$ characterization of the deposited $\mathrm{CsPbI}_{2.5} \mathrm{Br}_{0.5}$ thin film and $\mathrm{CsPbI}_{2.5} \mathrm{Br}_{0.5} / \mathrm{FCQDs} \mathrm{GHJ}$ thin films on glass substrates. By formation of graded heterojunction, the $\mathrm{PL}$ emission peak changed from $\sim 1.81 \mathrm{eV}$ to $\sim 1.82 \mathrm{eV}$ for the $\mathrm{CsPbI}_{2.5} \mathrm{Br}_{0.5}$ perovskite thin film. A blue-shifted PL emission peak can be correlated with filling of the trap states on $\mathrm{CsPbI}_{2.5} \mathrm{Br}_{0.5}$ layers' surface or along the grain boundaries by injection of FCQDs [40]. Moreover, a strong photoluminescence quenching of $\sim 60 \%$ was observed in the $\mathrm{CsPbI}_{2.5} \mathrm{Br}_{0.5} /$ FCQDs GHJ thin film as a result of charge transfer from $\mathrm{CsPbI}_{2.5} \mathrm{Br}_{0.5}$ to FCQDs. This was further confirmed by 


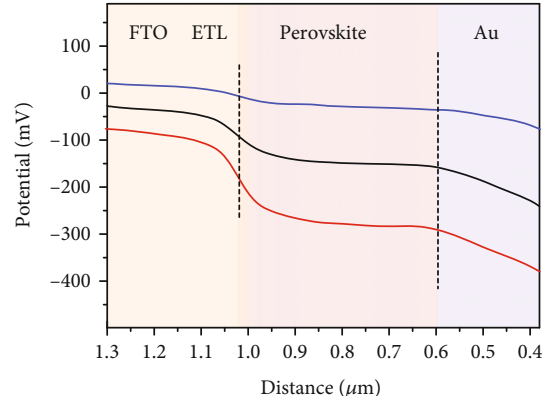

(a)

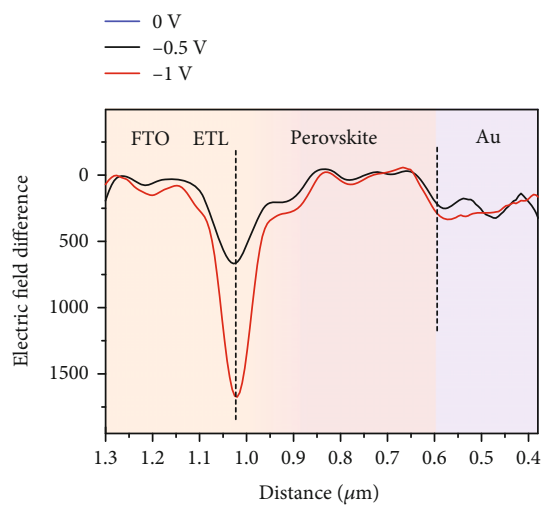

(c)

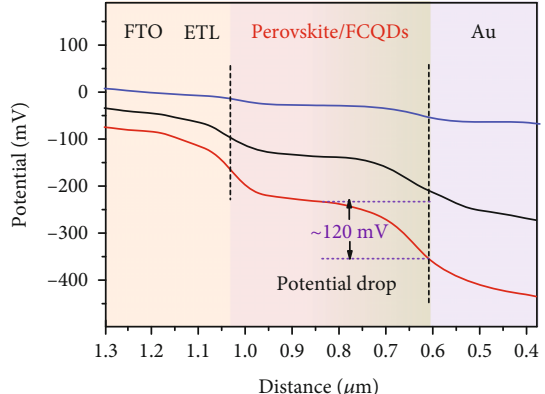

(b)

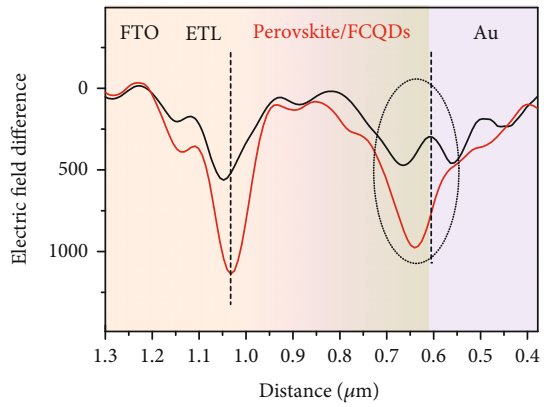

(d)

$$
--0.5 \mathrm{~V}
$$

FIGURE 2: Scanning Kevin probe force microscopy characterization of the graded heterojunction. Cross-sectional potential distribution of the samples $\left(\mathrm{FTO} / \mathrm{TiO}_{2} / \mathrm{CsPbI}_{2.5} \mathrm{Br}_{0.5} / \mathrm{Au}\right.$, sample (a), and $\mathrm{FTO} / \mathrm{TiO}_{2} / \mathrm{CsPbI}_{2.5} \mathrm{Br}_{0.5} / \mathrm{FCQDs} \mathrm{GHJ} / \mathrm{Au}$, sample (b)) under different bias voltages. The calculated electric field distribution of the corresponding samples under different bias voltages of the samples

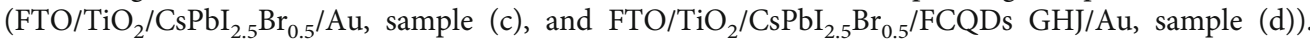

time-resolved PL decay characterization, Figure 1(e). A considerably reduced PL decay lifetime (from $\sim 21$ to $11 \mathrm{~ns}$ ) for the $\mathrm{CsPbI}_{2.5} \mathrm{Br}_{0.5} /$ FCQDs GHJ sample demonstrates substantial photogenerated charge carriers transfer from $\mathrm{CsPbI}_{2.5} \mathrm{Br}_{0.5}$ to FCQDs.

To gain more insight about the construction of $\mathrm{CsPb}_{2.5} \mathrm{Br}_{0.5} / \mathrm{FCQDs} \mathrm{GHJ}$ on charge transfer, we investigated the electrical potential distribution across the GHJ structure by performing scanning Kevin probe force microscopy (KPFM) characterization on cross-section of the $\mathrm{FTO} / \mathrm{TiO}_{2} / \mathrm{CsPbI}_{2.5} \mathrm{Br}_{0.5} / \mathrm{Au}$ and the $\mathrm{FTO} / \mathrm{TiO}_{2} / \mathrm{CsP}$ $\mathrm{bI}_{2.5} \mathrm{Br}_{0.5} / \mathrm{FCQDs} \mathrm{GHJ} / \mathrm{Au}$ samples in dark condition as shown in Figure S5 [41-44]. To exclude the interference of surface charges on the cleaved surface, the potential distribution measurements were performed at different biases voltage $(0 \mathrm{~V},-0.5 \mathrm{~V}$, and $-1.0 \mathrm{~V})$ [42]. Figures $2(\mathrm{a})$ and $2(\mathrm{~b})$ present the averaged potential profiles across the two samples. In contrast to the $\mathrm{FTO} / \mathrm{TiO}_{2} / \mathrm{CsPbI}_{2.5} \mathrm{Br}_{0.5} / \mathrm{Au}$ sample which exhibited a relatively constant potential distribution across the perovskite absorption layer, a potential drop of about $120 \mathrm{mV}$ was observed at the perovskite/FCQDs graded heterojunction area at $-1.0 \mathrm{~V}$ bias voltage in the $\mathrm{FTO} / \mathrm{TiO}_{2} / \mathrm{CsPbI}_{2.5} \mathrm{Br}_{0.5} / \mathrm{FCQDs} \mathrm{GHJ} / \mathrm{Au}$ sample. This obvious potential drop can be caused by charge diffusion motion at the perovskite/FCQDs interface, which also demonstrates the existence of depletion region at the perovskite/FCQDs heterojunction interface. The electric field distribution profiles can be determined as shown in Figures 2(c) and 2(d) by taking the first derivative of the potential differences (Figure S6) for the samples. For the control sample, the electric field at the perovskite/Au interface is quite weak, which implies no obvious depletion region at the interface. Therefore, the photo-generated holes need to diffuse through the whole perovskite absorption layer to be collected by the Au electrode. In contrast, a strong and wide nonzero electric field was identified at the perovskite/FCQDs graded heterojunction area, indicating the formation of a wide depletion region with strong built-in electric field across the junction. This wide depletion region across the perovskite/FCQDs graded heterojunction is expected to facilitate photogenerated hole extraction and thus decrease charge carrier recombination loss.

We further fabricated $\mathrm{CsPbI}_{2.5} \mathrm{Br}_{0.5} / \mathrm{FCQDs}$ GHJ PSCs using low-temperature processed carbon electrode as current collector. With the merit of low-temperature large-area processability and low manufacture cost, the carbon electrode based $\mathrm{CsPbI}_{2.5} \mathrm{Br}_{0.5} /$ FCQDs GHJ PSCs can be easily scaled up for practical applications. Figure 3(a) presents the crosssectional SEM image of a completed perovskite/FCQDs GHJ device, from which a dense and uniform $\mathrm{CsPbI}_{2.5} \mathrm{Br}_{0.5} / \mathrm{FCQDs}$ film can be clearly observed. Figure 3(b) 


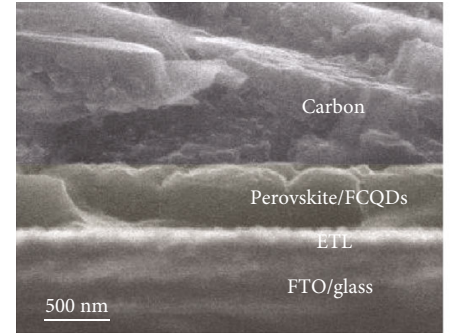

(a)

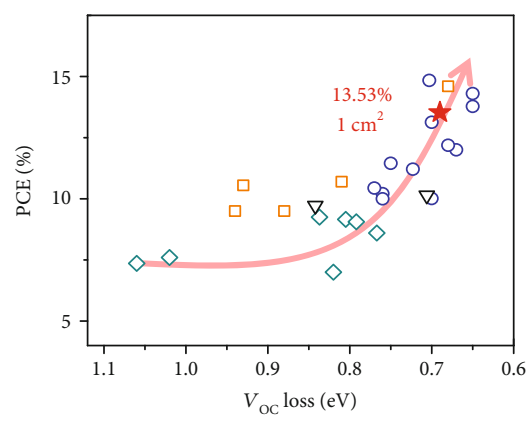

(c)

$$
\begin{aligned}
& \square \mathrm{CsPbI}_{3} \text { based } \\
& \bigcirc \mathrm{CsPbI}_{2} \mathrm{Br} \text { based } \\
& \diamond \mathrm{CsPbIB}_{2} \text { based }
\end{aligned}
$$

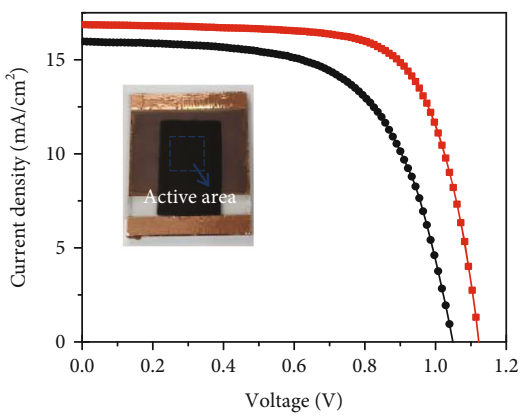

(b)

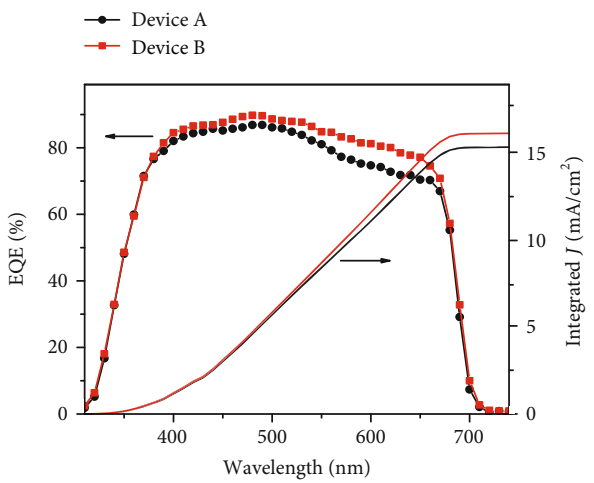

(d)

FIgURE 3: Photovoltaic performance of the graded heterojunction devices. (a) Cross-sectional SEM image of FTO/TiO $/ 2 / \mathrm{CsPb}_{2.5} \mathrm{Br}_{0.5} / \mathrm{FCQDs}$ $\mathrm{GHJ} /$ carbon completed perovskite device. (b) Current density-Voltage measurements of the $1.0 \mathrm{~cm}^{2}$ perovskite devices. The inset shows an optical photograph of a perovskite device. (c) A brief summary of perovskite devices that using inorganic perovskites as a light absorber and carbon counter-electrode as current collector reported so far. (Values of $V_{\mathrm{OC}}$ loss and PCE are taken from literature, Table S1, Supporting Information). (d) EQE spectra and integrated current density of the perovskite devices.

presents photocurrent density-voltage $(J-V)$ measurement curves of the optimized $1.0 \mathrm{~cm}^{2}$ PSC devices. We observed that the PSC device (device B) based on perovskite/FCQDs GHJ exhibited improved photovoltaic performance. The champion device showed a $V_{\mathrm{OC}}$ of $1.12 \mathrm{~V}$, a $J_{\mathrm{SC}}$ of $16.87 \mathrm{~mA} \mathrm{~cm}^{-2}$, a FF of $71.6 \%$, yielding an impressive PCE of $13.53 \%$. This is one of the highest PCE among carbon electrode-based inorganic PSCs reported so far. In contrast, the control device (device A) showed a total PCE of $10.70 \%$, with a $V_{\mathrm{OC}}$ of $1.05 \mathrm{~V}$, a $J_{\mathrm{SC}}$ of $15.97 \mathrm{~mA} \mathrm{~cm}^{-2}$, and a FF of $63.8 \%$. Figure 3(c) presents the plot of PCE versus $V_{\text {OC }}$ loss for currently reported PSCs (detail data can be found in Table S1) that using inorganic perovskites $\left(\mathrm{CsPbI}_{3}, \mathrm{CsPbI}_{2} \mathrm{Br}, \mathrm{CsPbIBr}_{2}\right.$, and $\left.\mathrm{CsPbBr}_{3}\right)$ as light absorber and carbon counter electrode as current collector. Apparently, our present fabricated $\mathrm{CsPbI}_{2.5} \mathrm{Br}_{0.5} /$ FCQDs GHJ device exhibited both high PCE and low $V_{\text {OC }}$ loss. This result substantiates the promising potential for constructing perovskite/FCQDs GHJ toward highly efficient PSCs. To the most of our knowledge, this is the first report of $1 \mathrm{~cm}^{2}$ carbon counter electrode based inorganic PSC with PCE exceeding 13\%. Figure 3(d) compares the results of external quantum efficiency (EQE) characterization of the aforementioned PSC devices.
Device A exhibited obvious EQE drop in long wavelength region $(>600 \mathrm{~nm})$, implying inefficient collection of charge carriers generated in the region close to the back contact of the absorber layer [45-47]. For the HTL-free PSCs, severe charge transfer barrier usually exists at the perovskite/counter electrode interface, thus decreasing charge collection efficiency in such region of the devices [45]. Impressively, device B based on perovskite/FCQDs $\mathrm{GHJ}$ exhibited much-improved EQE response in the long wavelength region, which confirms the augmented charge collection efficiency. The integrated $J_{\mathrm{SC}}$ calculated from the EQE spectra for devices A and B were estimated to be 15.40 and $16.19 \mathrm{~mA} \mathrm{~cm}^{-2}$, respectively.

Electronic impedance spectroscopy (EIS) measurements were conducted to understand the effect of perovskite/ FCQDs GHJ on electronic processes at the perovskite/carbon electrode interface. The measurements were performed by connecting the FTO glass as working electrode and carbon electrode as counter electrode and reference electrode [48-52]. Figure 4(a) presents the result of EIS characterization in the form of Nyquist plots when the devices were biased at $0.9 \mathrm{~V}$ under $10 \mathrm{~mW} \mathrm{~cm}^{-2}$ illumination. Two semicircles were observed in the frequency analysis. The first arc corresponds to charge transport processes in the 


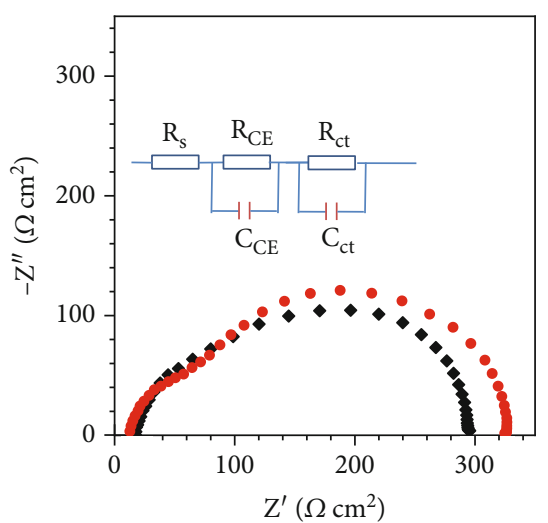

(a)

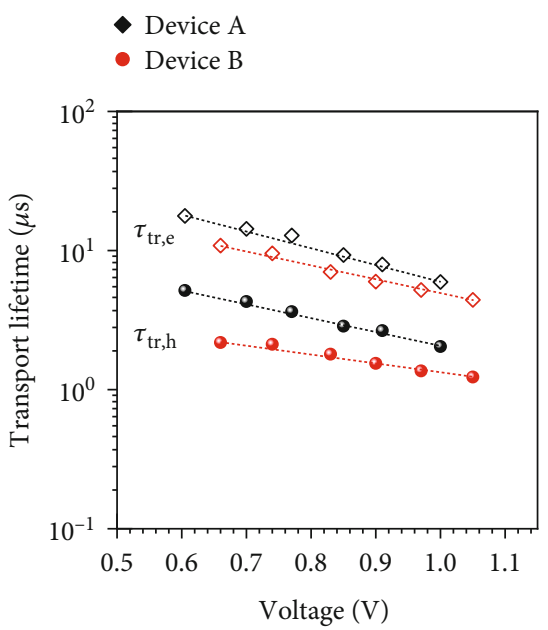

(d)

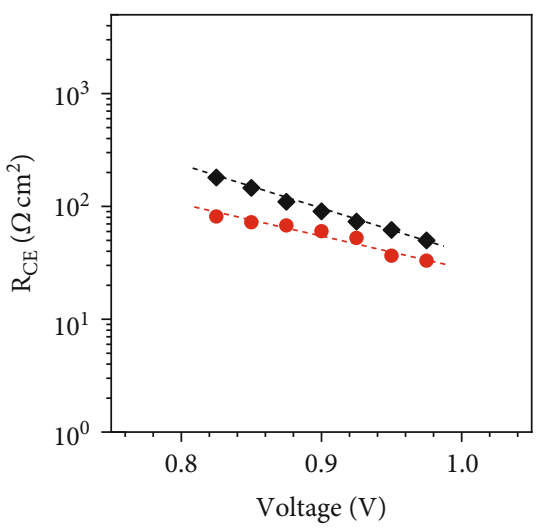

(b)

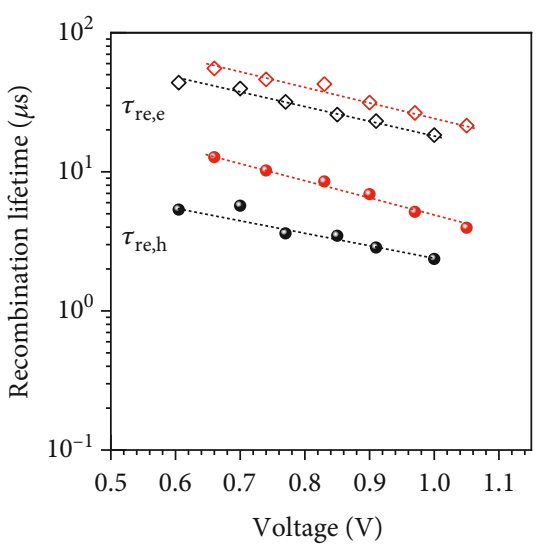

(e)

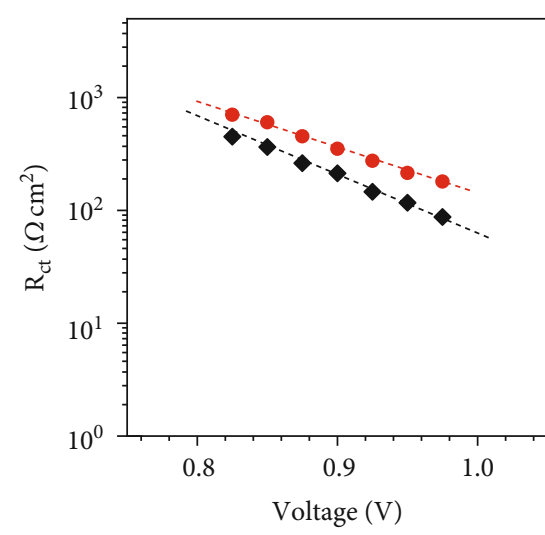

(c)

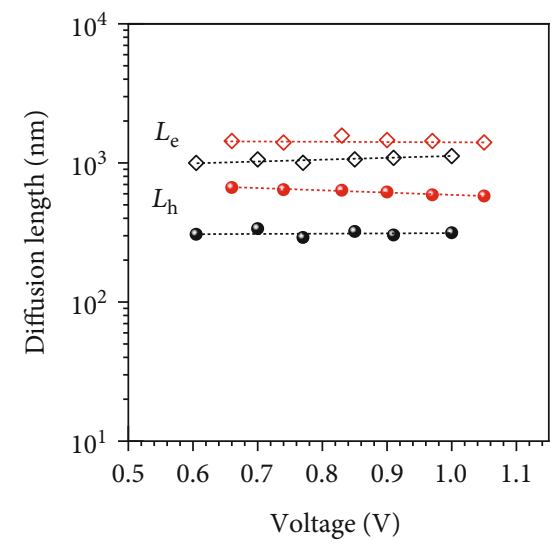

(f)

$\diamond$ Device A

- Device B

FIGURE 4: Interfacial charge transfer kinetics of the graded heterojunction devices. (a) Electronic impedance spectroscopy measurements of the devices under $10 \mathrm{mWcm}^{-2}$ illumination at a bias voltage of $0.9 \mathrm{~V}$. (b) Charge transport resistance $\left(R_{\mathrm{CE}}\right)$ and $(\mathrm{c})$ charge recombination resistance $\left(R_{\mathrm{CT}}\right)$ as a function of the applied bias voltage obtained from impedance measurements. (d) Transport lifetime $\left(\tau_{\mathrm{tr}}\right)$ and $(\mathrm{e})$ recombination lifetime $\left(\tau_{\mathrm{re}}\right)$ of the devices obtained from transient photovoltage/photocurrent decay measurements. (f) The calculated diffusion length of devices.

charge transport layers or at the charge transport layer/ counter-electrode interfaces in the high-frequency region, i.e., fast charge transport processes. The second arc can be assigned to charge recombination processes at the perovskite layer/charge selective contact interfaces in the intermediate frequency region, i.e., relative slow charge transport processes [49]. We fitted the EIS plots with equivalent circuit exhibited in the inset of Figure 4(a). Figure $4(\mathrm{~b})$ shows that device $\mathrm{B}$ presents smaller chargetransfer resistance $\left(R_{\mathrm{CE}}\right)$ at the perovskite layer/counterelectrode interface, which indicates faster transport of holes from the perovskite absorber to the carbon electrode. Figure 4(c) presents the fitted interfacial charge recombination resistance $\left(R_{\mathrm{CT}}\right)$ as a function of bias voltage. Apparently, device $\mathrm{B}$ exhibits larger $R_{\mathrm{CT}}$ under a series of bias voltage, indicating a slower interfacial charge recombination process. Moreover, a relatively smaller capacitance was calculated for device $\mathrm{B}$ from the response in the intermediate frequency region (Figure S7), implying less charge carrier accumulation at the perovskite layer/carbon electrode interface and thus the restrain of interfacial charge recombination [52].

To examine the effect of perovskite/FCQDs GHJ on dynamics of charge carrier transfer and recombination, the PSC devices were further characterized with transient photocurrent/photovoltage decay (TPC/TPV) measurements at various bias light intensities (Figure S8). The working principles for measurements of the TPC/TPV have been described in our previous reports [53-55]. By varying the bias light intensity, the corresponding recombination lifetime $\left(\tau_{\text {re }}\right)$ at a different open-circuit voltage (or quasiFermi level) can be obtained in the model of TPV. Similarly, the apparent transport lifetime $\left(\tau_{\text {tr }}\right)$ is collected at short circuit conditions in TPC measurements. The transport/recombination kinetics in PSCs are frequently modeled with biphasic decay (Figure S8), corresponding to 


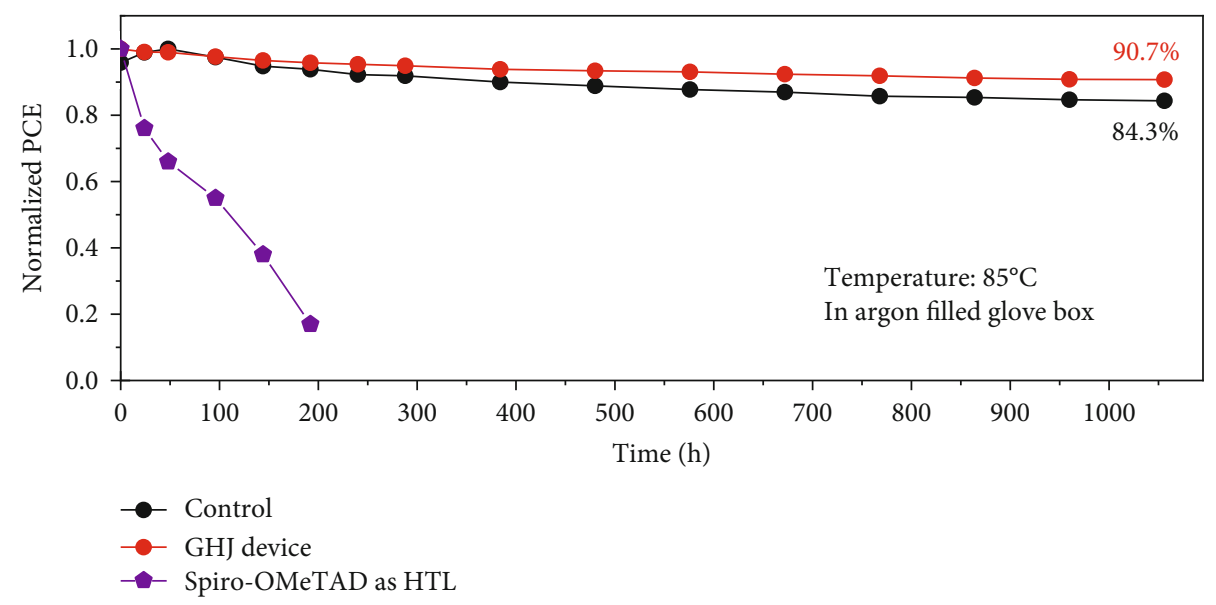

FIGURE 5: Stability of the graded heterojunction devices. Long-term stability test of unencapsulated PSC devices under $85^{\circ} \mathrm{C}$ thermal aging in argon-filled atmosphere.

the dynamics of electron and hole, respectively. Figure 4(d) exhibits the carrier transport lifetime as a function of $V_{\mathrm{OC}}$ (determined by the incident light intensity) for the devices by fitting the TPC curves. Notably, both electron and hole transport lifetimes of device B are significantly shorter than that of device A. At an identical voltage of $\sim 0.9 \mathrm{~V}$, the transport lifetime of electron $\left(\tau_{\text {tree }}\right)$ of device $\mathrm{B}$ is approximately $6 \mu \mathrm{s}$, being shorter than that of device A $(\sim 8 \mu \mathrm{s})$. Similarly, the transport lifetime of hole $\left(\tau_{\mathrm{tr}, \mathrm{h}}\right)$ of device $\mathrm{B}$ is approximately $1.5 \mu \mathrm{s}$, being shorter than that of device A $(\sim 2.6 \mu \mathrm{s})$. This result can be attributed to the $\mathrm{CsPb}_{2.5} \mathrm{Br}_{0.5} / \mathrm{FCQDs}_{\mathrm{GHJ}}$, which introduces additional driving force for hole transfer, therefore an enhanced charge carrier transport. This result also indicates an imbalanced charge transport in both devices. A similar behavior has been found in the TPV measurements (Figure 4(e)), showing that device B exhibited a longer carrier recombination lifetime $\left(\tau_{\text {re }}\right)$ of electron and hole compared to device A. At an identical voltage of $\sim 0.9 \mathrm{~V}$, the recombination lifetime of electron $\left(\tau_{\mathrm{re}, \mathrm{e}}\right)$ of device $\mathrm{B}$ is approximately $31.5 \mu \mathrm{s}$, being longer than that of device A $(\sim 23 \mu \mathrm{s})$. Similarly, the recombination lifetime of the hole $\left(\tau_{\mathrm{re}, \mathrm{h}}\right)$ of device B is approximately $7 \mu \mathrm{s}$, being longer than that of device A $(\sim 2.9 \mu \mathrm{s})$. A long interfacial recombination lifetime guarantees efficient charge collection and thus enhancing the device output photovoltage. The diffusion length $(L)$ of charge carriers in $\mathrm{CsPbI}_{2.5} \mathrm{Br}_{0.5}$ PSCs was further evaluated by the ratio of recombination lifetime and transport lifetime, as given by the expression $L=$ $\left(D \times \tau_{\text {re }}\right)^{1 / 2}$, where $D$ is the charge carrier diffusion coefficient obtained from charge transport time $\tau_{\text {tr }}$. As shown in Figure 4(f), device B has a longer charge diffusion length $(L)$. At an identical voltage of $\sim 0.9 \mathrm{~V}$, the diffusion length of electron $\left(L_{e}\right)$ of device B is approximately $1.46 \mu \mathrm{m}$, being longer than that of device $\mathrm{A}(\sim 1.06 \mu \mathrm{m})$. The diffusion length of the hole $\left(L_{h}\right)$ of device B is approximately $617 \mathrm{~nm}$, being longer than that of device $\mathrm{A}(\sim 303 \mathrm{~nm})$. In comparison with previous report on $\mathrm{CsPbI}_{2} \mathrm{Br}$-based inorganic PSC showing diffusion length of $463 \mathrm{~nm}$ for electrons and $202 \mathrm{~nm}$ for holes, in this study, the $\mathrm{CsPbI}_{2.5} \mathrm{Br}_{0.5} /$ FCQDs GHJ device exhibited much longer diffusion length for both electrons and holes [55]. Especially, such a greatly increased hole diffusion length indicates effective hole collection efficiency, which principally delivers higher output photocurrent. This result also well explains the increased $\mathrm{EQE}$ value observed for device $B$ in long wavelength region (Figure 3(d)), which can be ascribed to the better charge carrier collection efficiency. The negative slope of the EQE curve for both devices towards longer wavelengths could be due to the loss of carriers by recombination in the bulk of the perovskite layer. Clearly, we should concern to increase the hole diffusion length along with a reduced interfacial charge recombination with the aim to a balanced charge transport and further improvement in device performace.

Long-term stability is another momentous metrics for the practical application of PSCs [56]. To estimate longterm stability of the carbon electrode-based $\mathrm{CsPbI}_{2.5} \mathrm{Br}_{0.5}$ perovskite/FCQDs GHJ PSCs, the unencapsulated $\mathrm{CsPbI}_{2.5} \mathrm{Br}_{0.5}$ devices (control) and $\mathrm{CsPbI}_{2.5} \mathrm{Br}_{0.5} / \mathrm{FCQDs}$ $\mathrm{GHJ}$ devices were measured under $85^{\circ} \mathrm{C}$ thermal-aging condition in argon-filled glove box. Herein, $85^{\circ} \mathrm{C}$ was selected for thermal-aging according to the standard IEC 61215-1:2016 [57], and the inert atmosphere (argon) was chosen to investigate the intrinsic stability of PSCs by eliminating the interference of other stresses from the ambient $[58,59]$. The PSC device based on commonly used spiro-OMeTAD as HTL and Au as counterelectrode was measured under identical conditions as a reference. As shown in Figure 5, after thermal aging at $85^{\circ} \mathrm{C}$ for 1056 hours, the carbon electrode-based CsPbI ${ }_{2.5} \mathrm{Br}_{0.5}$ devices (control) maintained $84.3 \%$ of the initial efficiency, while the $\mathrm{CsPbI}_{2.5} \mathrm{Br}_{0.5} / \mathrm{FCQDs} \mathrm{GHJ}$ device maintained $90.7 \%$ of the initial efficiency. In contrast, the PSC device using spiro-OMeTAD as HTL and $\mathrm{Au}$ as counter-electrode almost lose $100 \%$ of the initial efficiency within 200 hours. The considerable improvement in long-term thermal stability of the carbon electrode-based $\mathrm{CsPbI}_{2.5} \mathrm{Br}_{0.5} / \mathrm{FCQD}$ GHJ devices can be attributed to the elimination of organic HTL and metal- 
electrode, as well as the robust shielding effect of FCQDs. These results demonstrate the significance of constructing carbon electrode-based inorganic perovskite/FCQDs GHJ PSCs for achieving long-term stability and provide important direction to realize low cost and highly stable inorganic PSCs toward commercialization.

\section{Discussion}

In summary, we first present a compositionally graded heterojunction structure for photovoltaic application by injecting fluorine-doped carbon quantum dots into inorganic perovskite $\mathrm{CsPbI}_{2.5} \mathrm{Br}_{0.5}$. This $\mathrm{CsPbI}_{2.5} \mathrm{Br}_{0.5} / \mathrm{FCQDs} \mathrm{GHJ}$ can effectively facilitate the separation of photo-generated charge carriers and their transport in photovoltaic devices. Using this novel inorganic perovskite/FCQDs graded heterojunction in conjunction with low-temperature processed carbon electrode, a PCE of $13.53 \%$ can be achieved over $1 \mathrm{~cm}^{2}$ device. Moreover, the carbon electrode based $\mathrm{CsPbI}_{2.5} \mathrm{Br}_{0.5} / \mathrm{FCQDs}$ GHJ PSCs exhibited excellent long-term stability, maintaining $90.7 \%$ of the initial efficiency under $85^{\circ} \mathrm{C}$ thermal aging for 1056 hours. This inorganic perovskite/FCQDs graded heterojunction offers a visible pathway to solve charge extraction obstacle in large-area carbon electrode based inorganic PSCs and provides an important direction to realize lowcost and highly stable inorganic PSCs toward commercialization.

\section{Materials and Methods}

Synthesis of fluorine-doped carbon quantum dots (FCQDs): a one-step hydrothermal method was adapted to prepare FCQDs [60]. Briefly, $360 \mathrm{mg}$ of citric acid, $1080 \mathrm{mg}$ of urea, and $200 \mathrm{mg}$ of sodium fluoride were added in $20 \mathrm{~mL}$ deionized water. The mixture was sonicated for about $30 \mathrm{~min}$ and transferred into a Teflon-lined autoclave. After heating at $180^{\circ} \mathrm{C}$ for $10 \mathrm{~h}$ and naturally cooling, the obtained FCQDs were purified by dialysis against distilled water. Then, the solution was centrifuged at $10000 \mathrm{rpm}$, and the obtained FCQDs were dispersed in chlorobenzene for use.

PSC devices fabrication: the compact $\mathrm{TiO}_{2}$ layer was prepared by spray pyrolysis deposition of titanium isopropoxide ethyl alcohol solution, followed by annealing at $100^{\circ} \mathrm{C}$ for 10 min and $450^{\circ} \mathrm{C}$ for $30 \mathrm{~min}$. The mesoporous $\mathrm{TiO}_{2}$ layer was prepared by spin-coating of $18 \mathrm{~nm}$ particle size paste (Dyesol 18 NR-D) diluted in ethanol at 3000 for rpm $30 \mathrm{~s}$, then sintered at $100^{\circ} \mathrm{C}$ for $10 \mathrm{~min}$ and $450^{\circ} \mathrm{C}$ for 1 hour. The $0.8 \mathrm{M}$ $\mathrm{CsPbI}_{2.5} \mathrm{Br}_{0.5}$ perovskite precursor solution was made by dissolving $\mathrm{CsI}, \mathrm{CsBr}$, and $\mathrm{PbI}_{2}$ (molar ratio $1: 1: 2$ ) in a mixture of DMF and DMSO $(v / v, 4: 1)$ and stirred overnight. For the graded heterojunction device, $2 \mathrm{mg} / \mathrm{mL}$ FCQDs were added into chlorobenzene antisolvent and stirred overnight. The perovskite solution was spin-coated onto $\mathrm{FTO} / \mathrm{TiO}_{2}$ substrates at $2000 \mathrm{rpm}$ for $10 \mathrm{~s}$ and $6000 \mathrm{rpm} 30 \mathrm{~s}$, and chlorobenzene with or without FCQDs was dropped onto the film at the last $23 \mathrm{~s}$ during the second step. Subsequently, the spin-coated perovskite films were dried for $5 \mathrm{~min}$, then thermally annealed at $70^{\circ} \mathrm{C}$ for $3 \mathrm{~min}$ and $300^{\circ} \mathrm{C}$ for $10 \mathrm{~min}$.
Finally, the carbon paste was coated by the doctor-blade method and annealed at $100^{\circ} \mathrm{C}$ for $30 \mathrm{~min}$ [61].

Other experimental details and characterizations are shown in the Supporting Information.

\section{Data Availability}

The experimental data is available if required.

\section{Conflicts of Interest}

The authors declare that there is no conflict of interest regarding the publication of this article.

\section{Authors' Contributions}

Q.S. and M.W. conceived the ideas. Q.S. conducted the device fabrication, performed the data analysis, and prepared the manuscript. C.S. and D.W. helped with the scanning KPFM characterization. Y.S. and X.Z helped with data analysis. T.Z. and H.B. helped with the device measurements. Z.Z. helped with the TOF-SIMS characterization. G.Y. provided the carbon paste. M.W. guided and supervised the whole study. All authors have given approval to the final version of the manuscript.

\section{Acknowledgments}

The authors thank the Analytical and Testing Centre of Huazhong University of Science \& Technology and the Nanodevices and Characterization Centre of WNLO-HUST for the measurements of the samples. This work was supported by the National Key Research and Development Program of China [2018YFB1502900, 2019YFE0101300], the National Natural Science Foundation of China [No. 21975088], and the National Natural Science Foundation of China Major International (Regional) Joint Research Project [NO.51961165106].

\section{Supplementary Materials}

Materials and experimental details are given in supporting information. Figure S1: (a) the chemical structure of fluorine-doped carbon quantum dots (FCQDs). (b) TEM and HRTEM (inset) images of FCQDs. (c) Diameter distribution of the presynthesized FCQDs from the TEM image measured using the Nano Measurer software. (d) XPS survey spectra of FCQDs. (e) Detailed C $1 \mathrm{~s}$ XPS spectra of FCQDs. (f) Detailed F $1 \mathrm{~s}$ XPS spectra of FCQDs. Figure S2: the derived curves from UV-vis absorption spectra for (a) FCQDs and (b) $\mathrm{CsPbI}_{2.5} \mathrm{Br}_{0.5}$ perovskite films for bandgap evaluation. (c) The curves of ultraviolet photoelectron spectroscopy (UPS) measurement on $\mathrm{CsPbI}_{2.5} \mathrm{Br}_{0.5}$ perovskite and FCQDs samples. (d) The calculated $E_{\mathrm{VB}}, E_{\mathrm{CB}}$ and $E_{F}$ of $\mathrm{CsPbI}{ }_{2.5} \mathrm{Br}_{0.5}$ perovskite and FCQDs. Figure S3: SEM images of control perovskite thin film and perovskite/FCQDs GHJ thin film. Figure S4: Pb 4f XPS spectra of control perovskite thin film and perovskite/FCQDs GHJ thin film. Figure S5: schematic diagram of scanning Kevin probe force microscopy characterization. Figure S6: calculated potential 
differences of the samples with respect to the $0 \mathrm{~V}$ curve. Figure S7: corresponding capacitance (C) as a function of the applied voltage at open-circuit condition obtained from impedance measurements. Figure S8: transient photocurrent and transient photovoltage decay curves of the devices under varying light intensity. The solid lines are fitted curves using a biexponential equation. Figure S9: current density-voltage measurement curves and PCE distribution of carbon electrode-based $\mathrm{CsPbI}_{2.5} \mathrm{Br}_{0.5} / \mathrm{FCQDs}$ planar heterojunction PSCs. Table S1: a brief summary of perovskite solar cells that using inorganic perovskites as light absorber and carbon counter electrode as current collector. (Supplementary Materials)

\section{References}

[1] W. Yang, B. Park, E. Jung et al., "Iodide management in formamidinium-lead-halide-based perovskite layers for efficient solar cells," Science, vol. 356, no. 6345, pp. 1376-1379, 2017.

[2] Q. Jiang, Y. Zhao, X. Zhang et al., "Surface passivation of perovskite film for efficient solar cells," Nature Photonics, vol. 13, no. 7, pp. 460-466, 2019.

[3] H. Min, M. Kim, S. Lee et al., "Efficient, stable solar cells by using inherent bandgap of $\alpha$-phase formamidinium lead iodide," Science, vol. 366, no. 6466, pp. 749-753, 2019.

[4] E. Alharbi, M. Dar, N. Arora et al., "Perovskite solar cells yielding reproducible photovoltage of $1.20 \mathrm{~V}$," Research, vol. 2019, article 8474698, 9 pages, 2019.

[5] J. Yoo, G. Seo, M. Chua et al., "Efficient perovskite solar cells via improved carrier management," Nature, vol. 590, pp. 587-593, 2021.

[6] Z. Wang, Z. Shi, T. Li, Y. Chen, and W. Huang, "Stability of perovskite solar cells: a prospective on the substitution of the A cation and X anion," Angewandte Chemie International Edition, vol. 56, no. 5, pp. 1190-1212, 2017.

[7] L. Chao, T. Niu, H. Gu et al., "Origin of high efficiency and long-term stability in ionic liquid perovskite photovoltaic," Research, vol. 2020, article 2616345, 13 pages, 2020.

[8] T. Miyasaka, A. Kulkarni, G. Kim, S. Öz, and A. Jena, "Perovskite solar cells: can we go organic-free, lead-free, and dopant-free?," Advanced Energy Materials, vol. 10, no. 13, article 1902500, 2020.

[9] J. Liang, Z. Liu, L. Qiu et al., "Enhancing optical, electronic, crystalline, and morphological properties of cesium lead halide by Mn substitution for high-stability all-inorganic perovskite solar cells with carbon electrodes," Advanced Energy Materials, vol. 8, no. 20, article 1800504, 2018.

[10] W. Zhu, Q. Zhang, D. Chen et al., "Intermolecular exchange boosts efficiency of air-stable, carbon-based all-inorganic planar CsPbIBr 2 Perovskite solar cells to over 9\%," Advanced Energy Materials, vol. 8, no. 30, article 1802080, 2018.

[11] C. Dong, X. Han, W. Li, Q. Qiu, and J. Wang, “Anti-solvent assisted multi-step deposition for efficient and stable carbonbased $\mathrm{CsPbI}_{2} \mathrm{Br}$ all-inorganic perovskite solar cell," Nano Energy, vol. 59, pp. 553-559, 2019.

[12] Z. Guo, S. Teo, Z. Xu et al., "Achievable high $V_{\text {oc }}$ of carbon based all-inorganic $\mathrm{CsPbIr}_{2}$ perovskite solar cells through interface engineering," Journal of Materials Chemistry A, vol. 7, no. 3, pp. 1227-1232, 2019.

[13] J. Liang, X. Han, J. Yang et al., "Defect-engineering-enabled high-efficiency all-inorganic perovskite solar cells," Advanced Materials, vol. 31, no. 51, article 1903448, 2019.
[14] H. Wang, H. Liu, Z. Dong, W. Li, L. Zhu, and H. Chen, “Composition manipulation boosts the efficiency of carbon-based $\mathrm{CsPbI}_{3}$ perovskite solar cells to beyond 14\%," Nano Energy, vol. 84, article 105881, 2021.

[15] S. Liu, L. Guan, T. Zhang et al., "Stable and efficient fullprintable solar cells using inorganic metal oxide framework and inorganic perovskites," Applied Materials Today, vol. 20, article 100644, 2020.

[16] J. Zhang, G. Hodes, Z. Jin, and S. Liu, "All-inorganic CsPbX Perovskite solar cells: progress and prospects," Angewandte Chemie International Edition, vol. 58, no. 44, pp. $15596-$ 15618, 2019.

[17] W. Chen, S. Zhang, Z. Liu et al., "A tailored nickel oxide holetransporting layer to improve the long-term thermal stability of inorganic perovskite solar cells," Solar RRL, vol. 3, no. 11, article 1900346, 2019.

[18] Q. Sun, G. Liang, X. Zhang, and M. Wang, "Sn/Pb binary metal inorganic perovskite: a true material worthy of trust for efficient and stable photovoltaic application," Science Bulletin, vol. 65, no. 16, pp. 1330-1333, 2020.

[19] H. Wang, H. Liu, W. Li, L. Zhu, and H. Chen, "Inorganic perovskite solar cells based on carbon electrodes," Nano Energy, vol. 77, article 105160, 2020.

[20] S. He, L. Qiu, D. Son et al., "Carbon-based electrode engineering boosts the efficiency of all low-temperature-processed perovskite solar cells," ACS Energy Letters, vol. 4, no. 9, pp. 2032-2039, 2019.

[21] S. Yoon, H. Min, J. Kim, G. Kim, K. Lee, and S. Seok, "Surface engineering of ambient-air-processed cesium lead triiodide layers for efficient solar cells," Joule, vol. 5, no. 1, pp. 183196, 2021.

[22] K. Cao, Z. Zuo, J. Cui et al., "Efficient screen printed perovskite solar cells based on mesoscopic $\mathrm{TiO}_{2} / \mathrm{Al}_{2} \mathrm{O}_{3} /$ $\mathrm{NiO}$ /carbon architecture," Nano Energy, vol. 17, pp. 171-179, 2015.

[23] X. Xu, Z. Liu, Z. Zuo et al., "Hole selective $\mathrm{NiO}$ contact for efficient perovskite solar cells with carbon electrode," Nano Letters, vol. 15, no. 4, pp. 2402-2408, 2015.

[24] F. Meng, A. Liu, L. Gao et al., "Current progress in interfacial engineering of carbon-based perovskite solar cells," Journal of Materials Chemistry A, vol. 7, no. 15, pp. 86908699, 2019.

[25] Z. Wu, Z. Liu, Z. Hu et al., "Highly efficient and stable perovskite solar cells via modification of energy levels at the perovskite/carbon electrode interface," Advanced Materials, vol. 31, no. 11, article 1804284, 2019.

[26] Y. Wu, X. Yang, W. Chen et al., "Perovskite solar cells with $18.21 \%$ efficiency and area over $1 \mathrm{~cm}^{2}$ fabricated by heterojunction engineering," Nature Energy, vol. 1, no. 11, article 16148, 2016.

[27] A. Rajagopal, P. Liang, C. Chueh, Z. Yang, and A. Jen, "Defect passivation via a graded fullerene heterojunction in lowbandgap Pb-Sn binary perovskite photovoltaics," ACS Energy Letters, vol. 2, no. 11, pp. 2531-2539, 2017.

[28] B. Li, Y. Zhang, L. Zhang, and L. Yin, "Graded heterojunction engineering for hole-conductor-free perovskite solar cells with high hole extraction efficiency and conductivity," Advanced Materials, vol. 29, no. 39, article 1701221, 2017.

[29] J. Han, X. Yin, Y. Zhou et al., "Perovskite/poly[bis(4-phenyl)(2,4,6-trimethylphenyl)amine] bulk heterojunction for high-efficient carbon-based large-area solar cells by gradient 
engineering," ACS Applied Materials \& Interfaces, vol. 10, no. 49 , pp. 42328-42334, 2018.

[30] Q. Zhao, A. Hazarika, X. Chen et al., "High efficiency perovskite quantum dot solar cells with charge separating heterostructure," Nature Communications, vol. 10, no. 1, p. 2842, 2019.

[31] S. Paulo, G. Stoica, W. Cambarau, E. Martinez-Ferrero, and E. Palomares, "Carbon quantum dots as new hole transport material for perovskite solar cells," Synthetic Metals, vol. 222, pp. 17-22, 2016.

[32] S. Zhou, R. Tang, and L. Yin, "Slow-photon-effect-induced photoelectrical-conversion efficiency enhancement for carbon-quantum-dot-sensitized inorganic CsPbBr3Inverse opal perovskite solar cells," Advanced Materials, vol. 29, no. 43, article 1703682, 2017.

[33] H. Hsu, H. Hsiao, T. Juang et al., "Carbon nanodot additives realize high-performance air-stable $\mathrm{p}$-i-n perovskite solar cells providing efficiencies of up to 20.2\%," Advanced Energy Materials, vol. 8, no. 34, article 1802323, 2018.

[34] Y. Ma, H. Zhang, Y. Zhang et al., "Enhancing the performance of inverted perovskite solar cells via grain boundary passivation with carbon quantum dots," ACS Applied Materials \& Interfaces, vol. 11, no. 3, pp. 3044-3052, 2019.

[35] S. Bai, P. Da, C. Li et al., "Planar perovskite solar cells with long-term stability using ionic liquid additives," Nature, vol. 571, no. 7764, pp. 245-250, 2019.

[36] K. Liu, S. Chen, J. Wu et al., "Fullerene derivative anchored $\mathrm{SnO}_{2}$ for high-performance perovskite solar cells," Energy \& Environmental Science, vol. 11, no. 12, pp. 3463-3471, 2018.

[37] Q. Sun, H. Li, X. Gong, H. Ban, Y. Shen, and M. Wang, "Interconnected SnO2Nanocrystals electron transport layer for highly efficient flexible perovskite solar cells," Solar RRL, vol. 4, no. 2, article 1900229, 2020.

[38] Y. Lv, H. Zhang, J. Wang et al., "All-in-one deposition to synergistically manipulate perovskite growth for highperformance solar cell," Research, vol. 2020, article 2763409, 10 pages, 2020.

[39] N. Li, S. Tao, Y. Chen et al., "Cation and anion immobilization through chemical bonding enhancement with fluorides for stable halide perovskite solar cells," Nature Energy, vol. 4, no. 5, pp. 408-415, 2019.

[40] H. Li, L. Tao, F. Huang et al., "Enhancing efficiency of perovskite solar cells via surface passivation with graphene oxide interlayer," ACS Applied Materials \& Interfaces, vol. 9, no. 44, pp. 38967-38976, 2017.

[41] B. Dänekamp, C. Müller, M. Sendner et al., "Perovskite-perovskite homojunctions via compositional doping," Journal of Physical Chemistry Letters, vol. 9, no. 11, pp. 2770-2775, 2018.

[42] P. Cui, D. Wei, J. Ji et al., "Planar p-n homojunction perovskite solar cells with efficiency exceeding 21.3\%," Nature Energy, vol. 4, no. 2, pp. 150-159, 2019.

[43] Y. Hou, E. Aydin, M. de Bastiani et al., "Efficient tandem solar cells with solution-processed perovskite on textured crystalline silicon," Science, vol. 367, no. 6482, pp. 11351140, 2020.

[44] C. Xiao, Q. Zhao, C. Jiang et al., "Perovskite quantum dot solar cells: mapping interfacial energetics for improving charge separation," Nano Energy, vol. 78, article 105319, 2020.

[45] H. Wei, J. Shi, X. Xu et al., "Enhanced charge collection with ultrathin $\mathrm{AlO}_{\mathrm{x}}$ electron blocking layer for hole-transporting material-free perovskite solar cell," Physical Chemistry Chemical Physics, vol. 17, no. 7, pp. 4937-4944, 2015.

[46] Y. Yu, C. Wang, C. Grice et al., "Synergistic effects of lead thiocyanate additive and solvent annealing on the performance of wide-bandgap perovskite solar cells," ACS Energy Letters, vol. 2, no. 5, pp. 1177-1182, 2017.

[47] Q. Sun, X. Gong, H. Li et al., "Direct formation of $\mathrm{I}_{3}{ }^{-}$ions in organic cation solution for efficient perovskite solar cells," Solar Energy Materials \& Solar Cells, vol. 185, pp. 111-116, 2018.

[48] M. Wang, P. Chen, R. Humphry-Baker, S. Zakeeruddin, and M. Grätzel, "The influence of charge transport and recombination on the performance of dye-sensitized solar cells," ChemPhysChem, vol. 10, no. 1, pp. 290-299, 2009.

[49] L. Zuo, H. Guo, D. deQuilettes et al., "Polymer-modified halide perovskite films for efficient and stable planar heterojunction solar cells," Science Advances, vol. 3, no. 8, article e1700106, 2017.

[50] X. Gong, Q. Sun, S. Liu et al., "Highly efficient perovskite solar cells with gradient bilayer electron transport materials," Nano Letters, vol. 18, no. 6, pp. 3969-3977, 2018.

[51] X. Shai, L. Zuo, P. Sun et al., "Efficient planar perovskite solar cells using halide Sr-substituted Pb perovskite," Nano Energy, vol. 36, pp. 213-222, 2017.

[52] H. Li, J. Lu, T. Zhang, Y. Shen, and M. Wang, "Cation-assisted restraint of a wide quantum well and interfacial charge accumulation in two-dimensional perovskites," ACS Energy Letters, vol. 3, no. 8, pp. 1815-1823, 2018.

[53] X. Zhao, S. Liu, H. Zhang et al., " $20 \%$ efficient perovskite solar cells with 2D electron transporting layer," Advanced Functional Materials, vol. 29, no. 4, article 1805168, 2019.

[54] H. Pan, X. Zhao, G. Xiu et al., "Controlling layered Ruddlesden-Popper perovskites via solvent additives," Nanoscale, vol. 12, no. 13, pp. 7330-7338, 2020.

[55] X. Gong, L. Guan, Q. Li et al., "Black phosphorus quantum dots in inorganic perovskite thin films for efficient photovoltaic application," Science Advances, vol. 6, no. 15, 2020.

[56] Q. Guo, J. Wu, Y. Yang et al., "High-performance and hysteresis-free perovskite solar cells based on rare-earthdoped $\mathrm{SnO}_{2}$ Mesoporous scaffold," Research, vol. 2019, article 4049793, 13 pages, 2019.

[57] "Terrestrial Photovoltaic (PV) Modules Design Qualification and Type Approval-Part 1: Test Requirements (IEC, 2016)," IEC 61215-1:2016.

[58] H. Snaith and P. Hacke, "Enabling reliability assessments of pre-commercial perovskite photovoltaics with lessons learned from industrial standards," Nature Energy, vol. 3, no. 6, pp. 459-465, 2018.

[59] S. Wu, R. Chen, S. Zhang et al., "A chemically inert bismuth interlayer enhances long-term stability of inverted perovskite solar cells," Nature Communications, vol. 10, no. 1, 2019.

[60] W. Yang, H. Zhang, J. Lai et al., "Carbon dots with red-shifted photoluminescence by fluorine doping for optical bio-imaging," Carbon, vol. 128, pp. 78-85, 2018.

[61] Q. Chu, B. Ding, J. Peng et al., "Highly stable carbon-based perovskite solar cell with a record efficiency of over $18 \%$ via hole transport engineering," Journal of Materials Science and Technology, vol. 35, no. 6, pp. 987-993, 2019. 